\title{
Essais
}

ESSAIS

Revue interdisciplinaire d'Humanités

$4 \mid 2014$

Éducation et humanisme

\section{Réflexions sur la relation entre littérature et nourriture dans l'œuvre de Yoko Tawada}

\section{Anne-Rose Meyer}

\section{(2) OpenEdition}

1 Journals

Édition électronique

URL : https://journals.openedition.org/essais/10104

DOI : 10.4000/essais.10104

ISSN : 2276-0970

Éditeur

École doctorale Montaigne Humanités

\section{Édition imprimée}

Date de publication : 15 avril 2014

Pagination : 159-173

ISBN : 978-2-9544269-2-1

ISSN : 2417-4211

\section{Référence électronique}

Anne-Rose Meyer, «Réflexions sur la relation entre littérature et nourriture dans l'œuvre de Yoko Tawada », Essais [En ligne], 4 | 2014, mis en ligne le 17 janvier 2022, consulté le 20 janvier 2022. URL http://journals.openedition.org/essais/10104 ; DOI : https://doi.org/10.4000/essais.10104 


\section{Réflexions sur la relation entre littérature et nourriture dans l'œuvre de Yoko Tawada}

\section{Anne-Rose Meyer}

Faire la connaissance d'une ville, d'une région, d'un pays, d'un peuple, cela signifie découvrir les trésors des musées, des monuments remarquables, des statues, la mode, le mode de vie et beaucoup d'autres choses, mais c'est finalement par la cuisine d'une région, d'un pays, qu'on expérimente le contact le plus direct. C'est une idée qui a inspiré de nombreuses recherches dans le domaine de l'ethnologie (Claude Lévi-Strauss) ${ }^{1}$. En ce qui concerne la littérature, la nourriture est un champ de recherche qui se développe depuis une vingtaine d'années ${ }^{2}$ - et je me propose d'examiner ce champ de recherche dont je pense que l'utilité et l'importance pour l'analyse de la littérature n'ont pas encore été entièrement inventoriées. Après une courte introduction, j'illustrerai la théorie par un exemple emprunté à la littérature allemande contemporaine en me référant à des textes de Yoko Tawada.

L'importance des besoins humains fondamentaux - se nourrir, boire - pour la formation d'une communauté est illustrée dans les textes les plus anciens de la littérature européenne. Dans L'Illiade et L'Odyssée on trouve maintes descrip-

1 Quelques indications bibliographiques : Claude Lévi-Strauss, Le Cru et le Cuit, Paris, Plon, 1964. Michael Andritzky (éd.), Oikos. Von der Feuerstelle zur Mikrowelle. Haushalt und Wohnen im Wandel, Gießen, Anabas, 1992. Eugene N. Anderson, Everyone Eats. Understanding Food \& Culture, New York, Londres, New York Univ. Press, 2005. Mary Douglas, Reinheit und Gefährdung. Eine Studie zu Vorstellungen von Verunreinigung und Tabu, trad. par Brigitte Luchesi, Berlin, Reimer, 1985. Mary Douglas, „Introduction. Some Unusual Ingredients and Possible Substitutes" in Jessica Kuper (éd.), The Anthropologists' Cookbook (Revised Edition), New York, Columbia University Press, 1997. Eleonore Schmitt, Das Essen in der Bibel. Literaturethnologische Aspekte, Münster, Lit, 1994.

2 Anne-Rose Meyer und Claudia Lillge (éd.), Interkulturelle Mablzeiten. Kulinarische Begegnungen und Kommunikation in der Literatur, Bielefeld, Transcript, 2004. Bernhard Wördehoff, „Sage mir, Muse vom Schmause... "Vom Essen und Trinken in der Weltliteratur, Darmstadt, Wissenschaftliche Buchgesellschaft, 2000. Alois Wierlacher, Vom Essen in der deutschen Literatur: Mablzeiten in Erzähltexten von Goethe bis Grass, Stuttgart, Kohlhammer, 1987. 
tions de fêtes, de sacrifices de vin et de chair pour les dieux. Plus tard, c'est dans le sacrifice de Jésus, lors de la célébration de la messe, que le vin et le pain jouent le rôle principal ${ }^{3}$. Au Moyen Âge, on connaît par exemple la technique de la ruminatio. Mâcher et remâcher les lettres, les phrases et les textes entiers sert à la remémoration, permet entre autres de s'identifier à la passion de Jésus ou des martyrs, d'apprendre par cœur des textes de la Bible ou des prières. Le lien entre les textes et la nourriture n'était pas compris autrefois en un sens figuré, mais en un sens littéral : la littérature est une nourriture spirituelle pour le croyant qui essaie d'incorporer son Dieu à d'autres moments que la messét. On pourrait parler encore longuement des aspects religieux de la nourriture qui est, au XVIII ${ }^{e}$ siècle, par exemple pour Friedrich Hölderlin dans son célèbre poème Brot und Wein, un moyen de distinguer mais aussi d'identifier l'Antiquité polythéiste et l'Antiquité monothéiste.

Si nous faisons défiler des siècles d'histoire littéraire, nous remarquons que manger et boire ont toujours été des thèmes principaux. Chez Balzac, par exemple, outre les scènes diverses qui se déroulent dans des restaurants parisiens ou en province, on pense à la faim qui sévit chez le père Goriot, on pense aux soirées, au thé dans les salons, etc. ${ }^{5} \mathrm{Et}$, des dizaines d'années plus tard, on pense - naturellement - à la gourmandise la plus connue dans notre contexte, à la douceur d'une madeleine dont l'arôme en harmonie avec le tilleul - une infusion - active toute la mémoire du narrateur. Ce sont ces arômes, ces odeurs qui réveillent en lui le royaume de son enfance et de sa jeunesse et on peut dire que cette expérience sensuelle sert - rétrospectivement - de moteur à la narration entière.

Quant à la littérature allemande, les scènes de table y ont toujours été au centre de processus communicatifs. Elles ont toujours offert à l'auteur la possibilité de présenter des caractères divers, de confronter des opinions, des modes de vie, des classes sociales. On rencontre par exemple dans Les Buddenbrook de Thomas Mann une série sans fin de dîners, de déjeuners, de petits-déjeuners pendant lesquels la famille et les invités conversent, discutent de questions diverses, se disputent, etc. Et des spécialités exotiques du nord de l'Allemagne sont évoquées - comme le Plettenpudding, terme qu'on ne peut guère traduire : le Plettenpudding est doux, mou, gras, trop sucré bien sûr et vraiment délicieux ${ }^{6}$ !

3 Jochen Hörisch, Brot und Wein: Die Poesie des Abendmahls, Francfort-sur-le-Main, Suhrkamp, 1992.

4 Caroline Bynum, Holy Feast and Holy Fast: the Religious Significance of Food to Medieval Women, Berkeley, Univ. of California Press, 1987.

5 Jutta Klose, Tafelfreud und Liebesleid in der Bourgeoisie: „Essen und Trinken“bei Balzac, Flaubert und Zola, Francfort-sur-le-Main, Peter Lang, 1987.

6 Michael Köhler, Götterspeise. Mahlzeitenmotivik in der Prosa Thomas Manns und Genealogie des alimentären Opfers, Tübingen, Niemeyer, 1996. 
Si l'on considère avec Wilhelm Voßkamp les textes littéraires comme " des phénomènes par lesquels une culture se reflète elle-même et parle d'ellemême $"{ }^{7}$ et si l'on analyse les valeurs symboliques de la nourriture et son importance pour la narration et pour la caractérisation des personnages, on est amené à distinguer deux aspects clefs, la représentation et l'incorporation. Ce sont les notions principales, qui intègrent le caractère performatif de boire et manger ainsi que la dimension esthétique de ces actes.

Manger et boire ne sont pas simplement un besoin naturel, ce sont aussi des phénomènes auxquels sont liées des idées fondamentales pour une culture. Le caractère barbare d'un peuple ou d'un groupe, par exemple, est souvent associé à ce qu'il mange : de la viande crue ? de quel animal ? du chien ? du cochon ? de la vache sacrée ? du lait tourné, autrement dit : du fromage ? Des catégories abstraites telles que riche/pauvre, propre/malpropre, sacré/profane, civilisél barbare, frais/pourri sont visualisées et valorisées par ce qu'une personne mange ou ne mange $\mathrm{pas}^{8}$. La nourriture permet de caractériser quelqu'un - un personnage fictif aussi - quant à son origine, son éducation, son salaire, sa couche sociale, son sens de la tradition, etc.

Les textes littéraires peuvent nous donner aussi des informations relatives aux tabous et aux préférences prédominantes dans une société ou un groupe : en effet, selon Wilhelm Voßkamp, on peut comprendre le système littéraire comme une partie du système de la société entière. Si l'on s'intéresse, même superficiellement, aux préférences culinaires des groupes, on voit que ce que les uns qualifient de délicieux, sain, prestigieux, luxueux, peut être inversement qualifié d'écœurant, pourri, impropre, dangereux en fonction des associations religieuses, culturelles ou mythiques, comme Roland Barthes l'avait déjà montré en 1957, dans ses Mythologies?. Manger et boire ne représentent pas seulement un besoin humain, en mangeant et en buvant on exprime aussi des valeurs fondamentales d'une société, certaines normes et un ordre symbolique. La nourriture et les scènes de table permettent à un auteur de marquer les différences qui existent entre sa propre culture et une culture dite étrangère. L'acceptation d'une culture étrangère peut par exemple être symbolisée dans un texte littéraire par des plats « exotiques » qu'un personnage sait savourer ${ }^{10}$ ou des rites qu'on accepte et imite.

7 Wilhelm Voßkamp, „Literaturwissenschaft als Kulturwissenschaft“, in Ansgar et Vera Nünning (éd.), Konzepte der Kulturwissenschaften. Theoretische Grundlagen - Ansätze - Perspektiven, Stuttgart, Weimar, Metzler, 2003, p. 73-85, ici p. 77, trad. Anne-Rose Meyer.

8 Le fait que la nourriture soit un symbole par lequel se constitue un ordre social, par exemple par les dichotomies mentionnées ci-dessus, est reconnu depuis 50 ans environ. L'ethnologie structuraliste, la philosophie, le folklore et l'anthropologie ont développé cette idée, voir les travaux de Claude Lévi-Strauss, Mary Douglas, Roland Barthes et Günter Wiegelmann. Un livre de Wiegelmann a inspiré en Allemagne la discussion et l'analyse de la nourriture sur le plan culturel : Günter Wiegelmann, Alltags- und Festspeisen. Innovationen, Strukturen und Regionen vom späten Mittelalter bis zum 20. Jahrhundert, Münster, New York, Munich, Waxmann, 1967.

9 Plus précisément dans ses essais « Le vin et le lait » et « Le bifteck et les frites ».

10 Voir l'introduction par Gerald Heidegger, „Essen und Charakter. Inszenierte Mahlzeiten in der 
Tous ces faits ont une certaine influence sur la méthode d'analyse. L'analyse ne peut pas être primordialement herméneutique, on a besoin d'une perspective interdisciplinaire - inspirée par la biologie, par exemple, ainsi que par la diététique, l'anthropologie, l'ethnologie, la philosophie, la pédagogie, la sociologie et la théologie.

La notion de "représentation " peut servir de fil conducteur à l'analyse. Elle met deux aspects en lumière : d'une part les techniques narratives, les symboles, les métaphores et autres formes d'images par lesquelles un auteur peut exprimer les différences entre groupes, puis leurs façons de se nourrir ; d'autre part, la notion de « représentation » renvoie au plan langagier, à la forme spécifique dans laquelle les denrées mentionnées sont décrites et liées à des rites, des distinctions sociales par exemple exprimées par le comportement à table, le plan de table, la hiérarchie des personnages à table, leur rang, la relation entre l'hôte et l'invité, la décoration, le bon goût et un «bon » comportement. Un fondement scientifique de cet aspect est fourni par la théorie de la civilisation de Norbert Elias et par les recherches de Pierre Bourdieu concernant le bon goût ${ }^{11}$.

L'analyse de la dimension représentative de manger et boire dans la littérature peut nous informer sur la façon dont l'auteur utilise des stéréotypes nationaux ou régionaux et caractérise ses personnages. Manger et boire servent souvent en littérature à établir des contrastes ou des correspondances entre les personnages.

Les denrées et la façon de les cuisiner sont souvent employées, en littérature, pour communiquer une impression de Heimat, de proximité ou de distance, ainsi que de métissage. Si on analyse les rôles que l'auteur donne à ses personnages masculins et féminins, on constate qu'il existe aussi une certaine tradition selon laquelle la viande est associée à l'homme ; manger de la viande exprime souvent une idée d'autorité masculine. Par contraste, la femme est associée à l'oikos, c'est-à-dire au foyer et à la maison où elle est responsable de la préparation de la nourriture ${ }^{12}$.

La notion de "représentation " intègre également l'expression des impressions sensuelles au sens de mimesis et imitatio, les cérémonies et les rites, l'étiquette (par exemple courtoise) qui règle le comportement et qui varie au cours du temps ${ }^{13}$.

Literatur“, in Hannes Etzelstorfer (éd.), Küchenkunst und Tafelkultur. Culinaria von der Antike bis zur Gegenwart. Mit 411 Abb., Vienne, Brandstätter, 2006, p. 377-388.

11 Norbert Elias, Überden ProzeßderZivilisation. Soziogenetische und psychogenetische Untersuchungen. 2 vol., Francfort-sur-le-Main, Suhrkamp, 1976. Pierre Bourdieu, La distinction. Critique sociale du jugement, Paris, Éd. de Minuit, 1979.

12 Alois Wierlacher, „Der ,wahre Feinschmecker" oder: Krieg und Frieden bei Tisch“, in Alois Wierlacher (éd.), Kulturthema Essen. Ansichten und Problemfelder, Berlin, Akademie Verlag, 1993, p. 279-287, ici p. 281.

13 Sur la représentation et l'étiquette on peut consulter Niklas Luhmann, Die Kunst der Gesellschaft, Francfort-sur-le-Main, Suhrkamp, 1995, voir p. 432 et 499 : „Das Kunstsystem 
La notion d' " incorporation " traite du corps d'un individu, car manger et boire sont des actes de passage, entre l'extérieur et l'intérieur, le " moi » et l'" autre ", qui se mêlent dans mon corps. On peut citer un essai de Sigmund Freud, Triebe und Triebschicksal (1915), à titre de référence pour ces phénomènes. Selon Freud, l'acte de manger est une forme ambivalente d'aimer, car cet amour anéantit l'existence indépendante de l'autre et symbolise par cela l'unité indissoluble de l'aimant et de l'être aimé ${ }^{14}$.

L'incorporation est un aspect intéressant, qui implique la discussion des questions et des phénomènes suivants :

- les métaphores (dévorer et manger) exprimant l'attraction sexuelle ou sa sublimation ;

- la fonction, l'importance et la portée des tabous concernant l'alimentation;

- la relativité des catégories comestible/non comestible à une époque où règnent la globalisation, le commerce mondial; où le goût individuel disparaît au profit d'un goût internationalisé par Lavazza, Nestlé, McDonald, Subway, etc. ;

- ne pas manger comme moyen de distinction, de prendre le dessus sur quelqu'un ou sur quelque chose ;

- les formes de l'abjection et sa relation avec la langue et l'art (Julia Kristeva et Winfried Menninghaus ${ }^{15}$ ) ; la question de ce que peut signifier le refus par un personnage de la nourriture offerte : la nausée marque-t-elle clairement une barrière entre le " moi » et les " autres", entre une culture et l'autre ?

- quelles sont les conséquences des idées de contamination et de nausée sur les notions d'identité personnelle et nationale ou régionale $?^{316}$

- si l'on arrive à surmonter le dégoût d'une certaine nourriture, est-ce que cela est l'expression d'un processus d'acculturation $?^{17}$

vollzieht Gesellschaft an sich selbst als exemplarischen Fall. Es zeigt, wie es ist." Voir aussi Jürgen Habermas, Strukturwandel der Öffentlichkeit, Francfort-sur-le-Main, Suhrkamp, 1990, p. 64 et 92, au sujet de la „Repräsentation als Demonstration von Bedeutsamkeit“. Cette forme de représentation se manifeste dans les fêtes, la danse, le théâtre, les tournois, les châteaux entourés de parcs, etc.

14 Sigmund Freud, „Triebe und Triebschicksale“, in Sigmund Freud, Gesammelte Werke. Chronologisch geordnet. Zehnter Band: Werke aus den Jahren 1913-1917, Francfort-sur-le-Main, Fischer, p. 209-232, ici p. 231.

15 Winfried Menninghaus, Ekel. Theorie und Geschichte einer starken Empfindung, Francfort-surle-Main, Suhrkamp, 2002, voir p. 25.

16 Voir Gisela Ecker, „,Fremdes Wasser'. Reisesteuerungen in Prosatexten der Gegenwartsliteratur“, in Anja K. Maier, Burkhardt Wolf (éd.), Wege des Kybernetes. Schreibpraktiken und Steuerungsmodelle von Politik, Reise, Migration, Münster, Lit, 2004, p. 218-234. Gisela Ecker, „Zuppa Inglese and Eating Up Italy. Intercultural Feasts and Fantasies", in Ralf Hertel, Manfred Pfister (éd.), Performing National Identity. Anglo-Italian Cultura Transactions, Amsterdam, Atlanta, Rodopi, 2008, p. 307-322.

17 Bernhard Waldenfels, Vielstimmigkeit der Rede. Studien zur Phänomenologie des Fremden 4, Francfort-sur-le-Main, Suhrkamp, 1999. 
De façon générale, l'analyse des repas et de la nourriture dans la littérature offre la possibilité de découvrir comment sont décrits la qualité d'étranger et a contrario les sentiments d'appartenance. La littérature sert d'archive en même temps qu'elle est un moyen pour exprimer des mémoires et des identités collectives ${ }^{18}$ - de sorte qu'on peut identifier en littérature les valeurs d'une société et l'ordre de la vie quotidienne. En tant qu'archive culturelle, la littérature nous donne la possibilité de repérer ces phénomènes au cours du temps et d'en faire une analyse diachronique. Elle nous rappelle que nos préférences et coutumes sont relatives, variables et diffèrent selon la culture ainsi que selon la société dans laquelle nous vivons ${ }^{19}$.

L'œuvre d'une auteure germano-japonaise, Yoko Tawada, montre la complexité de la relation entre littérature et nourriture. "C'est, dit-elle, une coutume étrange de manger avec le même organe avec lequel on parle $»^{20}$. Cette citation - empruntée à son premier livre, seulement là où tu es, là n'est rien - fait voir l'ambivalence de la bouche ${ }^{21}$ : elle forme un lieu de passage entre l'intérieur et l'extérieur, elle nous donne la possibilité d'incorporer quelque chose et d'exprimer quelque chose. La bouche et la langue - comme organe et comme métonymie pour la faculté de parler - sont des leitmotivs dans l'œuvre de Yoko Tawada ${ }^{22}$.

18 C'est le titre d'un essai écrit par Birgit Neumann, in Astrid Erll, Marion Gymnich, Ansgar Nünning (éd.), Literatur - Erinnerung - Identität. Theoriekonzeptionen und Fallstudien, Trier, WVT, 2003, p. 49-77.

19 J'ai développé ces réflexions concernant la représentation et l'incorporation avec Dr. Claudia Lillge, Université de Paderborn, voir Claudia Lillge, Anne-Rose Meyer, „Interkulturelle Dimensionen von Mahlzeiten", in Claudia Lillge, Anne-Rose Meyer (éd.), Interkulturelle Mablzeiten. Kulinarische Begegnungen und Kommunikation in der Literatur, Bielefeld, transcript, 2008, p. 11-22.

20 Yoko Tawada, nur da wo du bist da ist nichts. Traduit par Peter Pörtner. Tübingen, Konkursbuch, sans année de parution, p. 15 : „Überhaupt ist es eine seltsame Angewohnheit, mit demselben Organ Dinge zu verzehren, mit dem man Dinge sagt."

21 Thomas Sing parle de la bouche comme d'un „Symbol der Wahrheit und der Lüge, des Charakters, der Einverleibung und des Begehrens, des Lebens wie des Todes, einer nichtrationalen Kommunikation, des weibl. Geschlechts sowie der dichter. Rede“. Thomas Sing, „Mund“, in Günter Butzer, Joachim Jacob (éd.), Metzler Lexikon literarischer Symbole, Stuttgart, Weimar, Metzler, 2008, p. 239-240, ici p. 239.

22 Sur la notion de "langue " dans des contextes culturels et littéraires, voir l'introduction de Claudia Benthien, „Zwiespältige Zungen. Der Kampf um Lust und Macht im oralen Raum“, in Claudia Benthien, Christoph Wulf (éd.), Körperteile. Eine kulturelle Anatomie, Reinbek, Rowohlt, 2001, p. 104-132. 
Des interprètes telles que Sigrid G. Köhler, Mirjam Gebauer et Clara Ervedosa ont mis l'accent sur le lien important qui existe entre la langue et le langage $e^{23}$, mais le caractère sensuel de la nourriture dans l'œuvre de Yoko Tawada n'a pas été relevé.

Les personnages de Yoko Tawada sont marqués par la déterritorialisation. Ils se trouvent à Bochum, à Marseille, à Bordeaux ou dans des villes sans nom. En tout cas ils se trouvent toujours loin de leur patrie, de leur pays d'origine, qui n'est jamais décrit en détail : il s'agit du Vietnam ou - plus souvent - du Japon. L'auteure nous signale cependant, par la description de la tradition culinaire, que les racines de l'un des personnages se trouvent dans un pays d'Asie. Dans ses textes fantastiques ou irréels, elle nous donne aussi des impressions sensuelles et réalistes en thématisant l'absorption de nourriture et des repas.

C'est le cas dans le récit L'CEil nu. Dans ce texte, une jeune femme vietnamienne fait une conférence à Berlin-Est, elle y rencontre un étudiant, Jörg, venant d'Allemagne de l'Ouest. Jörg l'emmène à Bochum. Elle le quitte, se rend à Paris où elle vit clandestinement pendant des années chez des compatriotes et chez des Français. Le passage suivant est situé à Bochum où la protagoniste vit avec Jörg. Comme la jeune femme ne sait pas l'allemand, ils communiquent en russe. Jörg demande :

- Qu'est-ce que tu veux manger?

- Dupho.

- Du quoi ?

- Une espèce de nouilles.

- Précise.

Incapable que j'étais d'expliquer en russe qu'il fallait cuire de fines et longues nouilles de riz dans de l'eau, ajouter du citron vert, des pousses de soja crues, de la citronnelle, du piment et du plantain avant d'arroser le tout d'un bouillon, je résumai la recette sommairement : "Une soupe, des nouilles et des légumes crus, tout mélangé. " Jörg sortit et je retombai sur le lit [...].

À mon réveil, le repas était sur la table. C'était exactement ce que j’avais dit, mais chaque ingrédient était servi à part. Les nouilles étaient épaisses et molles, barbouillées de sauce tomate sanguinolente. La soupe était trop salée et couverte d'yeux de graisse. En garniture, de la laitue fripée et des tomates mayonnaise pas mûres ${ }^{24}$.

23 Sur la notion de langue dans l'œuvre de Yoko Tawada voir Sigrid G. Köhler, Körper mit Gesicht. Rhetorische Performanz und postkoloniale Repräsentation in der Literatur am Ende des 20. Jahrhunderts, Köln, Böhlau, 2006. Mirjam Gebauer, „'Lebensgeschichte einer Zunge'. Autobiographisches Schreiben jenseits der Muttersprache bei Yoko Tawada“, in Michael Grote, Beatrice Sandberg (éd.), Autobiographisches Schreiben in der deutschsprachigen Gegenwartsliteratur, t. 3 : Entwicklungen, Kontexte, Grenzgänge. Munich, Iudicium, 2009, p. 114-129. Clara Ervedosa, „,Europa mit der Zunge wahrnehmen`: Interkulturelle Begegnungen als ästhetisches Potential in den Texten Yoko Tawadas", in Jean-Marie Valentin (éd.), Akten des XI. Internationalen Germanistenkongresses Paris 2005, t. 6 : Migrations-, Emigrations- und Remigrationskulturen - Multikulturalität in der zeitgenössischen deutschsprachigen Literatur, Berne, Berlin, Bruxelles et al., Peter Lang, 2007, p. 341-348.

24 Yoko Tawada, L'Eil nu. Roman. Traduit de l'allemand par Bernard Banoun, Lagrasse, Verdier, 2005, p. 21. 
L'auteure utilise la description des repas pour illustrer des différences culturelles. La forme littéraire dans laquelle elle nous donne une impression de la nourriture est marquée par la dualité germano-vietnamienne. Le malentendu - le " pho " serait une somme d'éléments qu'on pourrait combiner librement - traduit la naïveté de Jörg en ce qui concerne non seulement la cuisine vietnamienne, mais la préparation des repas en général. Le plat cuisiné ne provoque donc chez la protagoniste qu'une sensation de nausée - au lieu d'appétit et de sentiments de gratitude.

Tawada ne fait pas voir cet aspect directement, par des commentaires critiques par exemple, mais par la perspective dans laquelle elle nous présente les aliments et par la description de leur qualité optique, leur fraîcheur et leur qualité gustative. Elle nous donne des impressions presque physiques et zoome sur les détails isolés, ce qui accentue le caractère réaliste du passage. Les nouilles décrites comme " grasses et pâles ", " saignantes de sauce tomate ", les " yeux " sur la soupe et la salade " ratatinée " nous font ressentir la nausée de la protagoniste. Et ce sentiment ne se réfère pas seulement au mets, mais aussi au personnage de Jörg qui est responsable de ce repas épouvantable.

Des émotions vives comme la nausée provoquent un moment de réflexion. Nous éprouvons du dégoût et un sentiment de rejet quand un élément est contraire à des valeurs essentielles de notre culture, quand nous craignons d'être contaminés par des ingrédients toxiques ${ }^{25}$. En thématisant cela, la narration de Tawada rend visible des catégories qui indiquent qu'un aliment est comestible : entre autres la fraîcheur, l'élasticité, un air appétissant et la " clarté ", une catégorie liée - selon Roland Barthes - à l'« idée d'une densité claire, d'une nutrition sans graisse, d'un élixir d'autant plus réconfortant qu'il est pur $»^{26}$.

Tawada montre au lecteur des plats européens par des vues prises de près et elle communique les réactions de sa protagoniste, c'est-à-dire sa nausée ou ses tentatives pour prendre ses distances par rapport aux repas offerts.

25 "La nausée sert à établir une limite, à rejeter quelque chose de dangereux, signaler une chose toxique ». Utz Jeggle, „Runterschlucken“, in Kursbuch, 29, 1997, p. 12-24, ici p. 14, trad. Anne-Rose Meyer.

26 Roland Barthes, L'Empire des signes, in Roland Barthes, Euvres complètes. Édition établie et présentée par Éric Marty, t. II, 1966-1973, Paris, Éditions du Seuil, 1994, p. 743-834, ici p. 754. 
La jeune femme pense à la cuisine de son pays, à la cuisine vietnamienne par exemple quand elle mange dans un restaurant italien à Bochum, quand elle fait la connaissance de Maria chez laquelle elle dort, quand elle prend le petit déjeuner français avec des compatriotes vietnamienne ${ }^{27}$ et finalement dans une vision du Banquet de Platon qu'elle imagine comme un dîner vietnamien :

J'enviais ces étudiants, mon vieux rêve me revenait à l'esprit : je voulais faire des études, étudier la philosophie [...].

Au lieu d'étudier, je passais mes journées à déambuler dans la ville. Sans un toit au-dessus de la tête, chômeuse clandestine, chose muette sans connaissances linguistiques, pas lavée et mal réveillée. Rue des Écoles, j’aperçus dans une vitrine un livre de Platon. J'ignorais tout de lui, mais ce nom m'était familier depuis l'enfance. Un camarade d'école [...] m'avait offert Le Banquet en édition de poche [...]. J'avais posé le livre sur mon bureau et tous les jours je regardais le titre. Le Banquet - je savais ce que ce mot signifiait et me représentais une vaste salle de fêtes ornée de lustres. Les grandes tables rondes étaient chargées de nouilles translucides, de crevettes roses, de pousses de soja, de coriandre et de citronnelle. Des régals roulés ou en boule remplissaient des jattes en porcelaine blanche et de grandes assiettes argentées. [...] Quelques femmes portaient l'ao dai, le vêtement traditionnel en soie plein de souplesse, d'autres d'élégantes robes du soir françaises. Elles allaient en cercles et marchaient d'un point à un autre comme dans une ronde, en quête de nouveaux interlocuteurs et de nouveaux verres de vin. C'était le banquet, et je n'en étais pas, j'étais dehors, dans la rue, l'éclat des lustres suintait par la fenêtre et projetait mon ombre sur les pavés ${ }^{28}$.

Dans l'Antiquité, des textes littéraires divers et des images sur des vases montrent que manger avec des hôtes et, ensuite, boire ensemble - le symposion - fut, des centaines d'années durant, une activité très appréciée. Le symposion rassemblait l'élite, le plus souvent les hommes. Les femmes de rang n'en faisaient partie qu'exceptionnellement, mais il y avait des musiciennes et des hétairres pour divertir la sociétée ${ }^{29}$. Le symposion donnait aux hommes la possibi-

27 Voir aussi Tawada, L'Eil nu, p. 18 : « Je sortis de la chambre et me rendis à la cuisine, où Jörg avait provoqué une espèce de gargouillis. [...] Dans le coin, une petite machine ventrue crachait dans son propre ventre transparent un liquide marron, c'était censé être du café, malgré l'apparence aqueuse et minable. [...] Tu ne veux vraiment pas de café ?

- Non! Je n'en veux pas, de ton café faiblard!

Si tu avais voulu m'offrir un café, il aurait fallu importer des grains de café vietnamien et les torréfier patiemment. Torréfier, torréfier, jusqu'à ce qu'ils commencent à dégager un parfum noir ébène. » Concernant le menu dans une pizzeria à Bochum la protagoniste dit : " La pizza avait un goût de vieux papier aromatisé à la tomate, et la boisson gazeuse rouge foncé celui d'un médicament contre les maux de gorge. J'aurais mille fois préféré de l'eau fraîche avec du citron vert et du sucre. [...] Tandis que nous découpions nos pizzas et mangions, moi, je partis dans des pensées de rouleaux de printemps vietnamiens aux herbes fraîches, au point d'en avoir l'eau à la bouche. » Sur les différences culinaires entre le Vietnam et la France et sur la nourriture voir p. 51 et 66 . La cuisine vietnamienne sert de référence positive. La cuisine européenne est déclarée immangeable.

28 Ibid., p. 151.

29 Voir Alfred Schäfer, Unterhaltungen beim griechischen Symposion: Darbietungen, Spiele und Wettkämpfe von homerischer bis in spätklassische Zeit, Mayence, von Zabern, 1997. 
lité d'échanger leurs opinions et en cela il avait aussi une dimension politique ${ }^{30}$. Sans s'attacher aux finesses textuelles des dialogues platoniciens, Yoko Tawada se réfere au texte de Platon et imagine le banquet comme une situation interculturelle dans laquelle on peut remarquer des influences françaises et des influences vietnamiennes. L'allusion à la coutume antique d'intégrer au symposion non seulement des délices sensuels mais aussi des discussions, des idées fait voir la situation précaire de la protagoniste dans L'CEil nu. En tant qu'immigrée illégale, elle est privée d'une formation régulière et vit à l'écart des couches sociales dans lesquelles elle pourrait se développer aussi sur le plan intellectuel, émotionnel et social. Dans les passages cités, mais aussi ailleurs dans le livre, l'expérience d'être exclu, de n'être d'aucun groupe est communiquée par des descriptions de nourriture. L'Eil nu est représentatif d'une technique narrative par laquelle Yoko Tawada traduit sa poétique du corps et des choses dans la représentation des repas - tout en soulignant leur matérialité pour décrire des relations complexes entre des cultures diverses - vietnamienne, française, allemande, italienne et, last but not least, antique et grecque.

\section{Plat principal : des œufs durs}

Yoko Tawada présente un autre aspect des repas interculturels dans son récit "Sans talon " [Fersenlos $]$. Dans ce récit, la protagoniste a dans sa poche trois œufs durs. Ce qui se passe est difficile à résumer, comme toujours chez Yoko Tawada, et le thème principal est lui aussi difficile à identifier. En fait, la protagoniste prend progressivement conscience du sentiment d'être mariée et de vivre avec son mari, elle essaie de s'intégrer tout en préservant sa propre identité.

La protagoniste, qui est en même temps la narratrice, est imaginée comme une étrangère arrivée en train d'un pays lointain qui n'est pas décrit. Dans sa poche, elle a entre autres " un stylo » et " trois carnets épais " par lesquels l'auteur la caractérise comme écrivaine. À part ces quelques objets, elle n'a presque pas de bagage - seulement des vêtements et ses trois œufs ${ }^{31}$. Les œufs sont un des motifs déterminants du récit. La narratrice voit régulièrement des images d'œufs dans la publicité. Arrivée à la maison de son mari, qu'elle ne connaît pas encore, elle reste seule. Le mari se cache, mais il fait la cuisine, aussi pour elle. Sa présence est indiquée par des traces, par des restes de repas :

30 Voir les essais dans l'anthologie éditée par Stefan Matuschek Wo das philosophische Gespräch ganz in Dichtung übergeht. Platons Symposium und seine Wirkung in der Renaissance, Romantik und Moderne, Heidelberg, Winter, 2002, en particulier Rainer Thiel, „Irrtum und Wahrheitsfindung. Überlegungen zur Argumentationsstruktur des platonischen Symposions“, op. cit., p. 5-16.

31 Yoko Tawada, "Fersenlos ", in Yoko Tawada, Tintenfisch auf Reisen. 3 Geschichten, trad. par Peter Pörtner, $2^{\mathrm{e}}$ édition remaniée, Tübingen, Konkursbuch, 1996, p. 9-76 (1 $1^{\mathrm{re}}$ édition, 1994), ici p. 9. 
il y a la table préparée pour le petit-déjeuner, avec un œuf dur intact et la coquille de l'œuf qu'il a mangés ${ }^{32}$. Les œufs sont ambivalents, en même temps concrets et symboliques :

Dans cette ville, il était important de manger les œufs verticalement. Pour cela, on a développé des ustensiles nommés coquetiers, car on ne doit pas manger les œufs horizontalement par erreur, m'avait expliqué quelqu'un dans le train, et quand je vis les coquetiers là sur la table, je pensai, c'est vraiment comme ça, et, pour enfreindre cette règle, je pris un œuf dans la main, m'imaginai qu'il serait le globe terrestre et le coupai - suivant l'équateur avec un couteau - en deux et mangeai le contenu. Puis, je réunis les deux moitiés de la coquille et les remis dans le coquetier, de sorte qu'on avait l'impression que le contenu était encore dedans. J'aurais pu dire que c'était une façon barbare de manger, mais j'étais seule et personne ne pouvait voir comment je mangeais et se vexer ou me corriger ou m'imiter ${ }^{33}$.

Manger est toujours lié à des valeurs culturelles et toujours influencé par des régulations régionales, comme le montre le passage cité ${ }^{34}$, ou comme le dit Alois Wierlacher : «La nourriture étant fondamentale pour la vie des hommes, les coutumes qui lui sont associées sont - en tant qu'éléments de la socialisation primaire - liées étroitement à notre système de communication, de sorte qu'on a caractérisé le fait de manger comme un "phénomène social total" "35.

La description des coutumes alimentaires offre la possibilité de caractériser des personnages. Le fait que la protagoniste du récit "Sans talons " vienne d'un pays asiatique est illustré par son étonnement en ouvrant le frigidaire où elle trouve divers produits laitiers qu'elle ne connaît pas ${ }^{36}$. Le comportement à table, la façon dont la protagoniste utilise les denrées révèlent des différences culturelles et la spécificité des rites. D'après Gian-Paolo Biasin : One of the most persistent forms of prejudice has to do with the attitude persons and peoples have toward the food of others - the choices of the foodstuffs to be cooked, the alimentary taboos, the ways of eating and drinking, the ways of speaking about what is eaten ${ }^{37}$.

32 Voir ibid., p. 23 et p. 32.

33 Ibid., p. 24.

34 Voir Claudia Lillge, Anne-Rose Meyer, "Interkulturelle Dimensionen von Mahlzeiten ", in Claudia Lillge, Anne-Rose Meyer (éd.), Interkulturelle Mahlzeiten. Kulinarische Begegnungen und Kommunikation in der Literatur, Bielefeld, transcript, 2008, p. 11-22.

35 Alois Wierlacher, Vom Essen in der deutschen Literatur: Mablzeiten in Erzähltexten von Goethe bis Grass, Stuttgart, Berlin, Mayence, Kohlhammer, 1987, p. 13.

36 Yoko Tawada, «Fersenlos», p. $21:$ : Des produits laitiers [...] que je n'avais jamais vus, du lait tourné épais, un yaourt qui n'était pas doux, le fromage blanc, un fromage bleu, un fromage sous forme de grains de riz, quand je me mis à manger, j'en étais obsédée, et mon mari me devint indifférent. Mon mari n'est qu'une partie du mariage, si j'habite cette maison pour de bon, si je mange chaque jour des produits laitiers et si - comme c'était écrit dans les papiers je peux fréquenter une école qui me plaît, il peut être comme il veut, pensai-je, et je me mis le dernier morceau de fromage dans la bouche. » Trad. A. Meyer.

37 Gian-Paolo Biasin, "Other Foods, Other Voices », in $M L N, 109,1994$, p. 831-846, ici p. 831. 
Yoko Tawada problématise ce fait - les différences qui existent entre les catégories comestible/non comestible dans les cultures - plusieurs fois dans son récit, ici par exemple :

[...] Je trouvai sur la table encore une fois des petits morceaux de coquille que je ramassai vite, mis dans la bouche et mangeai. Si j'expliquais à mon mari, dans mon pays on ne mange que la coquille et on jette le reste dans la poubelle, le calcium est bon pour les ongles et le reste ne contient que du cholestérol, il se fâcherait et dirait, encore un mensonge pour me tester, ou il crierait et dirait tu penses sûrement que ton mari est encore plus stupide que tu craignais ${ }^{38}$.

En outre, la qualité de ce texte réside dans l'art de faire voir - grâce à la description des gestes de la vie quotidienne et d'un régime spécial - la complexité des œufs. L'œuf et le "globe ", avec lequel elle compare l'œuf, ont comme trait commun d'être des systèmes fermés qui sont un abri pour la vie qui s'y développe pour un certain temps. Dans l'œuf, il y a au commencement du développement du poussin un dépôt d'énergie. L'œuf est cassé quand le poussin en sort, lorsque le dépôt est vidé. Ces aspects offrent la possibilité d'une autre interprétation de ce récit. La protagoniste incorpore aussi le monde de son mari lorsqu'elle casse la coquille et mange l'œuf. À la fin du récit, elle casse la porte de son bureau pour voir s'il n'est pas là, mais elle trouve seulement un calamar mort. On peut interpréter le texte comme le résultat de ce processus d'annexion. Il aurait ainsi une dimension métafictionnelle, ce que souligne la dernière phrase :

[...] si les connexions et complications étranges se résolvaient, un nouveau commencement devrait être possible, je pensais toujours je ne l'ai tout de même pas tué, je ne fis ouvrir la porte que pour récupérer mes œufs et mes carnets ${ }^{39}$.

Les œufs sont aussi dans "Sans talons " des symboles de fertilité correspondant à la grossesse, un thème qui apparaît plusieurs fois dans le texte et qui est lié étroitement à l'acte productif d'écrire, qui n'est jamais thématisé directement mais qui est présent - indirectement - par des objets tels que le stylo, l'encre, les carnets. On ne saurait produire des analogies plus claires en ce qui concerne les œufs. Ils correspondent aussi à l'acte de nourrir. Le mari toujours absent nourrit sa femme. Cette dernière fait la cuisine, ce qui est l'élément constitutif de leur vie :

Tout était calme dans la maison, mais les lumières étaient allumées, dans une casserole dans la cuisine, il y avait une soupe à la crème pour moi, plus tard, au lit, je décidai de faire moi-même la cuisine à partir de demain, comme il se doit pour une mère de famille, car si je vivais comme ça, comme à l'hôtel, je n'aurais jamais le sentiment de vivre mariée, avec ces pensées agréables je m’endormis ${ }^{40}$.

38 Yoko Tawada, "Fersenlos ", op. cit., p. 32.

39 Ibid., p. 74.

40 Ibid., p. 30-31. 
Par la préparation de la nourriture et par sa consommation, Yoko Tawada déstabilise non seulement la différence entre "l'intérieur " et "l'extérieur ", mais aussi entre "le moi " et "l'autre ». La préparation de la nourriture et sa consommation sont dans ce récit un acte communicatif. On peut penser à Freud qui, dans son essai Triebe und Triebschicksale (1915), affirme que manger ou dévorer est une sorte d'acte d'amour.

\section{Deuxième plat et dessert : moules et sucre}

On voit que le palais et la langue, " manger » et " parler " ne forment pas un système uniforme dans l'œuvre de Yoko Tawada, mais qu'il s'agit de phénomènes multifonctionnels et ambivalents. Cette impression est renforcée par le récit «La Bivalve », publié dans le livre ÜberSEEzungen, en 2002. Le titre du livre consiste en un jeu de mots : les notions Übersetzung (traduction) et Seezunge (sole) sont combinées. Le titre du récit " La Bivalve " se réfère aux moules - et à beaucoup d'autres choses.

Il s'agit d'un texte très structuré qui est divisé en deux parties : des scènes irréelles, comme celle présentée ci-dessous, sont suivies par des considérations de la narratrice concernant la ville de Marseille et ses sentiments durant son stage là-bas au "Centre de la poésie » - c'est la traduction littérale -, qui sont décrits d'une façon réaliste :

Le couple est assis à une table, qui se trouve dans la rue, devant un petit restaurant de poissons, et mange des moules. Un vent venant du port tourne les coins de la nappe, comme s'il y cherchait des secrets. Mes jambes sont attachées par une corde humide, je reste sous la table. [...] Les passantes ne font pas attention à moi. Mon corps leur fait probablement penser plutôt à un colis postal qu'à une sirène. Le couple gobe, mange bruyamment et pousse des gémissements. De temps en temps, il me jette des coquillages vides ${ }^{41}$.

Les moules sont dans ce texte une image traditionnelle de la sexualité féminine et forment un élément clef d'une scène sado-masochiste ${ }^{42}$. L'indice selon lequel la moule contient quelque chose, la chair, correspond à une symbolique ambivalente. D'un côté, il y a l'idée que le féminin, que le maternel est un récipient qui contient un élément vivant. D'un autre côté, le féminin, le maternel, peut aussi absorber une substance qui ne sera plus jamais libre. On peut dans ce contexte lire le fait que le corps féminin de la narratrice est lié par une corde humide comme un signe qui renvoie à la moule close, à

41 Yoko Tawada, „Die Zweischalige“, in Tawada, Yoko, ÜberSEEzungen. Tübingen, Konkursbuch, 2002, p. 36-41, ici p. 36, trad. A. Meyer.

42 Les moules peuvent être interprétées comme un symbole de vie pécheresse et d'avarice, ce qui correspond à la scène citée, si on pense au couple mangeant. Sur la moule comme symbole littéraire voir Dietmar Peil, „Muschel“, Metzler Lexikon literarischer Symbole, p. 240-241, ici p. 240. 
une sexualité restreinte et une "objectivation » de la femme comme " colis ». Le coquillage, ainsi que la mer, sont mentionnés plusieurs fois dans le passage cité, la mer est bien un des symboles du féminin et du maternel ${ }^{43}$.

Dans la scène du restaurant suit un commentaire neutre de la narratrice qui raconte son stage à Marseille : "On remarque aussitôt que les yeux de Marseille ville portuaire ont vu des centaines de figures étrangères. Je me sentis ici aussitôt détendue, j'étais libérée des regards sceptiques ou curieux des paysans, qui m'avaient accablée en province ${ }^{44}$. La technique littéraire consistant à faire suivre un passage irréel d'une séquence réaliste est caractéristique de tout le texte et de sa structure : "La Bivalve » n'est pas seulement le titre du récit, mais aussi un programme.

La continuité et la cohérence sont apportées par le fait qu'on a la même narratrice dans les deux parties de la narration. Par exemple, le couple finit son repas dans la deuxième partie irréelle, il emmène la narratrice malgré sa protestation : "Je dois travailler demain toute la journée au Centre de la poésie ". Il l'attache chez eux à une chaise. Dans une diction plus neutre, la narratrice décrit son travail au Centre de la poésie. Les descriptions de ce travail sont mélangées avec des descriptions de nourriture :

Nos textes furent traduits cru et cuit, et puis imités. [...] À midi, nous mangeâmes au restaurant dans la cour [...]. Dans chaque bouchée il y avait un espace fluide où se développait quelque chose de doux. Je ne veux pas dire un goût doux au sens propre, mais une acceptation du plaisir de la langue [Zungenlust]. À Hambourg, où j'habite, on déteste tout ce qui est "doux ». Là-bas, le crémant, le vin ou le café ne doivent pas - en aucun cas - être doux [...]. Pourquoi ont-ils peur du doux ? Un goût doux a-t-il peut-être un caractère puéril, superflu ou mensonger $?^{45}$

Le doux comme motif imprègne les scènes suivantes : le couple force la narratrice à lécher sa propre peau, et celle-ci est surprise que sa chair ait un goût si doux. Quand elle doit ramper dans une masse de déchets, elle dit à deux reprises que la masse est douce. Eros et la décadence, la perte d'identité et la découverte de son propre corps : ce sont les thèmes incontournables étroitement liés dans ce texte.

43 Cette connotation est connue aussi dans la culture japonaise, voir Manfred Lurker, „Muschel“, Wörterbuch der Symbolik, éd. par Manfred Lurker, 5édition augmentée, Stuttgart, Kröner, 1991, p. 496-497, ici p. 496. Voir aussi Manfred Lurker, „Meer“, op. cit., p. 471 et Hildegard Kretschmer, „Muschel“, Lexikon der Symbole und Attribute in der Kunst, éd. Hildegard Kretschmer, Stuttgart, Kröner, 2008, p. 290-291. Kretschmer voit dans le coquillage un symbole de la fécondité et de l'eau, ce qui prend forme concrète dans l'image de la naissance d'Aphrodite ou de Vénus. Ces déesses sortent de coquillages.

44 Yoko Tawada, „Die Zweischalige“, p. 36, trad. A. Meyer.

45 Ibid., p. 38. 
La structure du récit nous rappelle que la moule est en même temps - comme l'œuf dans le récit "Sans talons " - une chose concrète et un indice poétologique. Car la structure du récit « La Bivalve » est bivalve, et l'instance narratrice est ambiguë elle aussi. Elle est un médium qui reflète et commente des phénomènes culturels et est en même temps aussi présente dans sa sensualité et sa corporéité. "La Bivalve », en thématisant le "Centre de la poésie " et ses correspondances avec le thème de la douceur, est également un texte autoréférentiel par lequel Yoko Tawada nous donne une idée de l'origine corporelle et sensuelle sur laquelle naît la poésie.

En résumé : le thème de la nourriture lie le langage et le corps. Manger perd dans l'œuvre de Yoko Tawada son caractère quotidien et banal. Elle accentue l'importance du sapor, du goût, pour l'homo sapiens : selon Michel Serres, homo sapiens se réfère d'abord à celui qui a du goût, " bête à saveur, avant de vouloir dire l'homme devenu tel par jugement, intelligence ou sagesse [...] : la sagesse vient après le goût, elle ne peut advenir sans lui, mais l'oublie $»^{46}$.

Avoir actualisél'expérience corporelle et sensuelle de la bouche, l'absorption de nourriture et le goût comme base de la communication (inter-)culturelle, intellectuelle et esthétique est une des grandes qualités de Yoko Tawada.

Anne-Rose Meyer

Université de Hambourg anne-rose.meyer@uni-hamburg.de

\section{Résumé}

Après avoir rappelé la place que la thématique de la nourriture a de tout temps occupée dans la littérature et inventorié différents aspects et différentes approches de la relation entre littérature et nourriture, l'auteure de cet article illustre sa réflexion par des exemples empruntés aux récits de l'écrivaine germano-japonaise Yoko Tawada : Les aliments, leur préparation, consommation et absorption y servent de marqueurs ou de révélateurs des différences culturelles entre Extrême-Orient et Occident.

\section{Mots-clés}

Nourriture, littérature, différences culturelles, communication interculturelle, Tawada.

\section{Abstract}

Food and eating have always been given an important place in literature. There have been numerous theoretical approaches concerning food and, especially, food as a literary motif. These considerations are illustrated with examples taken from the stories of the Japanese-German writer Yoko Tawada, in which food, its preparation and absorption, are used as markers of cultural differences between the Far-East and the West.

\section{Keywords}

Food, literature, cultural differences, intercultural communication, Tawada.

46 Michel Serres, Les cinq sens. Philosophie des corps mêlés, I. Essai, Paris, Grasset et Fasquelle, 1985 , p. 167. 